\title{
Pattern of Bone Mineral Density (BMD) Among Patients Attending Tertiary Hospital: 9 years' Experience
}

\author{
Zeenat Jabin, Raihan Hussain, Shamim M.F. Begum, Rahima Parveen, Nasreen Sultana, Nurjahan Khatun
} National Institute of Nuclear Medicine \& Allied Science

Address for correspondence: Dr. Zeenat Jabin, Principal Medical Officer, National Institute of Nuclear Medicine \& Allied Sciences (NINMAS), Block-D, BSMMU Campus, Shahbagh, Dhaka, Email: zeenatjabin@yahoo.com

\section{ABSTRACT}

Objective: A retrospective study was conducted to assess the pattern of bone density status in a large population of both sexes who attended tertiary hospital National Institute of Nuclear Medicine and Allied Sciences (NINMAS) in a specific time period.

Materials and Methods: A retrospective study was carried out on 2777 patients who were referred to NINMAS for dual energy $X$ - ray absorptiometry (DXA) measurement of bone mineral density (BMD) during the year March 2005 to January 2014. BMD was measured at femoral neck (Ward's triangle) and lumbar spines with a Norland XR-36 machine. Data about age, sex, body weight, presentations, back pain status and menstrual history were recorded. Few of them had known osteoporosis and history of fracture. Reporting was done according to the T score following WHO criteria. Few reports were based on the Z-score.

Results: A total of $2777(\mathrm{M}=788, \mathrm{~F}=1989$ with a ratio of 1: 2.5) patients with age ranging from 12 to 85 (mean + SD $43 \pm 11.1)$. BMD findings and relevant history were recorded. Results showed normal bone density in 478 $(17.21 \%)$, osteopenia in $866(31.18 \%)$, osteoporosis in $1108(39.91 \%)$ and discrepancy of BMD in hip and spine in $325(11.70 \%)$ patients. Female patients $(n=1989)$ were sub divided into 6 sub groups according to age range and significant relationship of bone loss with pre peri and postmenopausal status of women were observed. These 6 groups included women of 25-34 years, 35-44years, 45-54 years, 55-64 years, 65-74 years and 75-85 years. Among them, the largest subgroups of $845(42.4 \%)$ were of 55-64 years and maximum number $(54.9 \%)$. of osteoporosis cases were of this age range. About $70 \%$ patients experienced low back pain with variable severity and duration. Mean body weight ( \pm SD) among the premenopausal women was $49.9 \pm 9.4 \mathrm{Kg}$ and $48.2 \pm 10.8$ $\mathrm{kg}$ in postmenopausal women.

Conclusion: The results of this study suggest that advancing age and menopausal condition of female and low body weight are important risk factors for the occurrence of low BMD.

Keywords: Dual energy $\mathrm{X}$ - ray absorptiometry (DXA), World Health Organization (WHO) criteria, $T$ score, Osteoporosis.

\section{INTRODUCTION}

Bone mineral density (BMD) measurements using dual energy X-ray absorptiometry (DXA) scans at the spine and hip have a significant role in the assessment of individuals at risk of osteoporosis. DXA bone densitometry studies provide useful information using World Health Organization (WHO) definitions of osteoporosis, osteopenia and normal bone density. Thus it plays major roles in the prediction of fracture risk and monitoring changes in BMD.

Compared with alternative bone densitometry techniques, hip and spine DXA examinations have a number of advantages that include interpretation using the WHO criteria, ability to predict fracture risk, proven effectiveness at targeting anti-fracture therapies and the ability to monitor response to treatment (1).

DXA uses two X-ray beams with different energy levels to determine the bone mineral content which is accomplished by subtracting the difference of absorption of X-rays between soft tissue and calcium of bone. The scanner software calculates the bone mineral content by the area of the region of interest (ROI). The BMD is compared to reference data specific to the scanner, and the results are expressed as the $\mathrm{T}$ score and the $\mathrm{Z}$ score and bone mineral contents in $\mathrm{gm} / \mathrm{cm}^{2}$ (2). The $\mathrm{T}$ score is the number of standard deviations above or below the mean value for young adult reference data which is considered to represent peak bone mass. The Z-score is the number of standard deviations below an average person of the same age (3). Identification of patients especially women with low BMD is an important strategy in reducing the incidence of osteoporotic fractures. However, screening of all women is not recommended by 
WHO (3). The WHO criteria list four diagnostic categories on the basis of the $\mathrm{T}$ score.

The present study was conducted to assess the bone density status among individuals of both sexes referred to NINMAS for DEXA scan during last 9 years and focused on the pattern of bone loss in different age groups especially women.

\section{MATERIALS AND METHODS}

This retrospective study was done among the patients referred to NINMAS during March 2005 to January 2014 for BMD measurements. Norland XR-36 machine was used to measure BMD in lumbar vertebrae (L1-L4) and femoral neck (Ward's triangle) in 2777 patients. DEXA was calibrated daily using a phantom provided by the manufacturer. Anthropometric measurements like age, sex, body weight, height were recorded in a light cotton dress designed for BMD studies. Clinical presentations of the patients, back pain status and menstrual history were recorded. Few of them had known osteoporosis and history of fracture. Reporting was done according to the WHO T-score criteria shown in Table-1 (3). No pediatric software was there in Norland XR-36 machine and in young patients less than 18 years; $\mathrm{Z}$ score was used for reporting.

Table 1: The World Health Organization (WHO) classification of BMD*

\begin{tabular}{|l|l|}
\hline \multicolumn{1}{|c|}{ Classification } & \multicolumn{1}{c|}{ T-score } \\
\hline Normal & -1.0 or greater \\
\hline Low Bone Mass (Osteopenia) & Between -1.0 and -2.5 \\
\hline Osteoporosis & -2.5 and below \\
\hline Severe Osteoporosis & $\begin{array}{l}-2.5 \text { and below with history of } \\
\text { fragility fracture }\end{array}$ \\
\hline \multicolumn{2}{|l}{${ }^{*}$ WHO Study Group 1994 } \\
\hline
\end{tabular}

\section{RESULTS}

A total of $2777(\mathrm{M}=788, \mathrm{~F}=1989$ with a ratio of 1 : 2.5) patients with age ranging from 12 to 85 years (mean + SD $43 \pm 11$.1years) have undergone BMD studies. BMD findings and relevant history were recorded. Results showed normal bone density in
478 (17.21\%), osteopenia in $866(31.18 \%)$, osteoporosis in 1108 (39.91\%) and discrepancy of BMD in hip and spine in $325(11.70 \%)$ patients. About 1989 female patients were sub divided into 6 groups according to age range and significant relationship of bone loss with pre, peri and postmenopausal status of women were observed.

Only 11 patients of 12 to 24 years were not included in a separate sub group as the number was less. Reporting of BMD was based on $\mathrm{Z}$ score in 6 patients who were less than 18 years of age. Female patients above 24 years were divided into 6 groups -those were of 25-34 years, 35-44years, 4554 years, 55-64 years, 65-74 years and 75-85 years. Among them, the largest subgroup of 845 $(42.4 \%)$ females was within 55-64 years and maximum number of osteoporosis 464 (54.9\%). Most of the osteoporosis cases belong to this age range. About $70 \%$ patients experienced low back pain with variable severity and duration. Mean body weight ( \pm SD) among the premenopausal women was $49.9 \pm 9.4 \mathrm{Kg}$ and $48.2 \pm 10.8 \mathrm{Kg}$ in postmenopausal women. Among the 788 male patients, 208 (26.4\%) were found osteoporotic, 260 (33\%) osteopenic and 97 (12.3\%) had normal bone mineral density. Discrepant findings in 223 male patients are not included in these percentage calculations.

Descriptive characteristics of the study population with results are summarized in Table: 2 and Figure-1.

Table 2: Pattern of BMD with descriptive characteristics of the study population $(n=2777)$

\begin{tabular}{|l|c|c|}
\hline \multicolumn{1}{|c|}{ Characteristic } & & Percentage \\
\hline Mean age & \multicolumn{1}{|c|}{ \pm 11.1 years } & \\
\hline Mean body weight ( \pm SD) Kg & $\begin{array}{l}49.9 \pm 9.4 \mathrm{Kg} \\
\text { (premenopausal) } \\
48.2 \pm 10.8 \mathrm{~kg} \\
\text { (postmenopausal) } \\
57.8 \pm 9.8 \mathrm{Kg}(\text { male) }\end{array}$ & \\
\hline Male: Female & $1: 2.5$ & \\
\hline Normal BMD & 478 & 17.21 \\
\hline Osteopenia & 866 & 31.18 \\
\hline Osteoporosis & 1108 & 39.91 \\
\hline $\begin{array}{l}\text { Discrepancy of hip and spine } \\
\text { BMD }\end{array}$ & 325 & 11.70 \\
\hline Back pain & 1943 & 70 \\
\hline Known H/O fracture & 229 & 8.2 \\
\hline
\end{tabular}




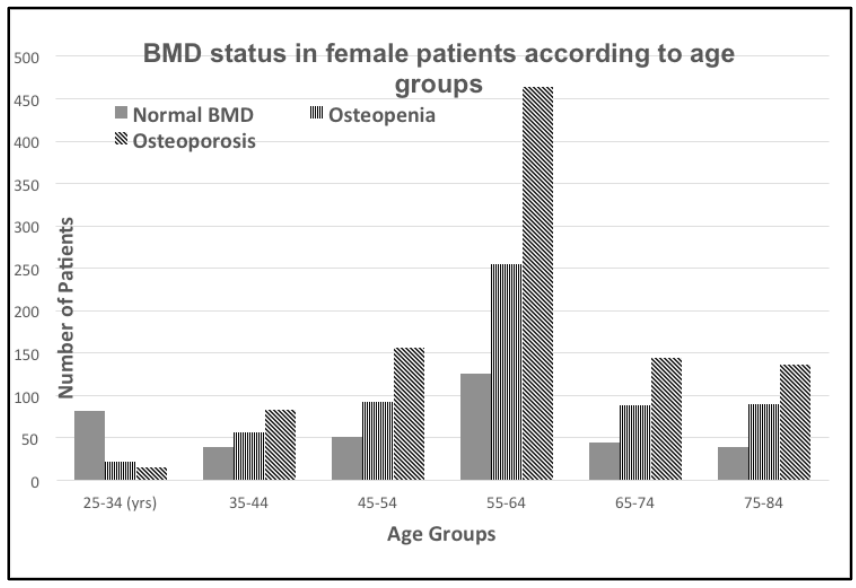

Figure 1- BMD status in female patients according to age groups $(n=1978)$

\section{DISCUSSION}

DXA is recognized as the reference method to measure BMD with acceptable accuracy errors and good precision and reproducibility. WHO has established DXA as the best densitometric technique for assessing BMD in postmenopausal women and based the definitions of osteopenia and osteoporosis on its results. DXA allows accurate diagnosis of osteoporosis, estimation of fracture risk and monitoring of patients undergoing treatment (4). Identification of patients especially women with low BMD is an important strategy in reducing the incidence of osteoporotic fractures. However, screening of all women is not recommended by WHO (3). Osteoporosis is a metabolic bone disorder characterized by low bone mass and microarchitectural deterioration, with a subsequent increase in bone fragility and susceptibility to fracture (1).

Last 9 years study at NINMAS revealed that BMD changes little during the early years of life and begins to decline with age and perimenopausal state with substantial decrease during the late perimenopause and early postmenopausal years. Our study showed, 15 out of 109 (13.7\%) women of 25-34 years had osteoporosis and this value increased to $46.3 \%$ (83 out of 179) immediately in the next age group of 35-44 years and further rise in percentage in 157 patients $(52.1 \%)$ is observed in the next age group 45-54 years. However, the incidence of osteoporosis was found maximum in women of 55-64 years in 464 (54.9\%) patients. We observed the peak BMD upto the age 34 years and then BMD declines gradually with aging. Average age of menopause in this population was 49.1 years. Joel S. Finkelstein et al. showed that BMD loss was fastest in women who became postmenopausal, next fastest in women who became late perimenopausal, and undetectable in pre- and early perimenopausal women (2). Youngest patient in this study was a girl of 12 years who had history of fracture with severe hypocalcaemia and was found to be osteoporotic. A 14-year-old boy was reported osteoporotic according to $\mathrm{Z}$ score who was a diagnosed case of SLE with nephritis, pulmonary TB and low serum calcium level.

Symptoms vary according to age, body weight, physical inactivity, menstrual status, chronic diseases, food habits etc. About 70\% patients experienced low back pain with variable severity and duration. About $16 \%$ patient had loss of height, which was mostly due to collapsed vertebra, kyphosis and stooped posture. We found that patients who are overweight had relatively better bone density than the low body weight patients of same age group.

Several studies demonstrated positive association of elevated body weight and/or BMI with bone mineral density or even protective effect against osteoporosis and fractures (5). Principal explanation of this association was heavier mechanical loading on bones with subsequent bone remodeling to resist this loading. Some of other putative mechanisms are increased estrogen synthesis (in women), increased secretion of insulin and increased plasma levels of leptin (6).

There were children among these 2777 patients and the lowest age recorded was 12 years. Most of them were referred with provisional diagnosis of metabolic diseases; unexplained hypocalcaemia, malabsorption syndromes and one had diagnosed osteogenesis imperfecta. There was no data for 
normal values in children of South Asian region and $\mathrm{T}$ score had little meaning for interpretation in these cases, since $\mathrm{T}$ scores are calculated for a peak bone mass that occurs between the ages of 20 to 30 years (4). We used $Z$ scores for this group of patients and reported accordingly. As children have different rates of growth, the first BMD report may be regarded as baseline with information of $\mathrm{Z}$ score and request the referring physician to further follow up.

Men also can have osteoporosis. Among the 788 male patients, 208 (26.4\%) were found osteoporotic, 260 (33\%) osteopenic and 97 $(12.3 \%)$ had normal bone mineral density. Discrepant findings were more evident in male patients than female and we found 223 males who had discrepancies in femur neck and lumbar spines $\mathrm{T}$ scores. The most frequent causes of low bone mineral density in men are idiopathic (35\% to 50\% of cases), alcoholism, steroid therapy and low testosterone levels. Smoking decreases bone mass in both men and women $(7,8)$. In osteoporosis, once a fracture occurs, the risk of a subsequent fracture is high. The best predictor of fracture is a previous fracture. Therefore, the diagnosis of osteoporosis should be made before the first fracture occurs, so that the patient can undertake lifestyle changes and undergo treatment to prevent fractures (6). Vertebral fractures can lead to severe chronic pain of neurogenic origin, which can be hard to control. Patients at high risk for fracture are most likely to benefit from therapy. Most Fractures Occur in Patients with T-score greater than -2.5 (9). The National Osteoporosis Foundation recommends drug therapy for osteoporosis in patients with $\mathrm{T}$ scores of -1.5 or lower who have other risk factors, and in patients with $\mathrm{T}$ scores of 2 or lower without other risk factors (8).

Limitations of this study were that BMI could not be correlated with BMD. Future studies on BMD correlation with BMI, detail history including calcium intake, physical activity, lifestyle, sun exposure, milk intake and parity of female patients are recommended.

\section{CONCLUSION}

This study was an attempt to identify one of the important public health problems like osteoporosis, which can be controlled if preventive measures are taken at an early stage. The results of this study suggest that advancing age and menopausal condition are an important risk factor for the occurrence of low BMD.

\section{REFERENCES}

1. Blake GM, Fogelman I. The role of DXA bone density scans in the diagnosis and treatment of osteoporosis. Postgrad Med J 2007; 83:509-517.

2. Blake GM, Fogelman I. Dual energy X-ray absorptiometry and its clinical applications. Semin Musculoskelet Radiol 2002; 6:207-18.

3. World Health Organization. Assessment of fracture risk and its application to screening for postmenopausal osteoporosis: technical report series 843. Geneva:WHO, 1994.

4. Magharaoi AE, Roux C. DXA scanning in clinical practice. Q J Med 2008; 101:605-617

5. Lloyd JT, Alley DE, Hawkes WG. Body mass index is positively associated with bone mineral density in US older adults. Arch Osteoporos. 2014 Dec; 9(1): 175.

6. Gonnelli S, Caffarelli C, Nuti R. Obesity and fracture risk. Clin Cases Miner Bone Metab. 2014 Jan; 11(1): 9-14.

7. Ross PD, Davis JW, Epstein RS, Wasnich RD. Pre-existing fractures and bone mass predict vertebral fracture incidence in women. Ann Intern Med 1991; 14:919-923.

8. National Osteoporosis Foundation. Physician's Guide to Prevention and Treatment of Osteoporosis; Washington, DC: National Osteoporosis Foundation; 2005.

9. De Laet CEDH, Van Hout BA, Burger H, et al. Hip fracture prediction in elderly men and women: validation in the Rotterdam study. J Bone Miner Res 1998;13:1587-1593. 